\title{
Diversity and Abundance of Arsenic Biotransformation Genes in Paddy Soils from Southern China
}

\author{
Si-Yu Zhang, ${ }^{\dagger \dagger}$ Fang-Jie Zhao, ${ }^{\S}, \|$ Guo-Xin Sun, ${ }^{\dagger}$ Jian-Qiang Su, ${ }^{\#}$ Xiao-Ru Yang, ${ }^{\#} \mathrm{Hu} \mathrm{Li}^{\text {, }}$ \\ and Yong-Guan $\mathrm{Zhu}^{*},+\#$
}
${ }^{\dagger}$ State Key Lab of Urban and Regional Ecology, Research Center for Eco-Environmental Sciences, Chinese Academy of Sciences, Beijing 100085, People's Republic of China
${ }^{\ddagger}$ University of Chinese Academy of Sciences, Beijing 100049, People’s Republic of China
$\S$ Jiangsu Collaborative Innovation Center for Solid Organic Waste Resource Utilization, College of Resources and Environmental Sciences, Nanjing Agricultural University, Nanjing 210095, People's Republic of China
"Sustainable Soil and Grassland Systems Department, Rothamsted Research, Harpenden, Hertfordshire AL5 2JQ U.K.
${ }^{\#}$ Key Laboratory of Urban Environment and Health, Institute of Urban Environment, Chinese Academy of Sciences, Xiamen 361021, People's Republic of China

\section{Supporting Information}

ABSTRACT: Microbe-mediated arsenic (As) biotransformation in paddy soils determines the fate of As in soils and its availability to rice plants, yet little is known about the microbial communities involved in As biotransformation. Here, we revealed wide distribution, high diversity, and abundance of arsenite (As(III)) oxidase genes (aio $A)$, respiratory arsenate $(\mathrm{As}(\mathrm{V}))$ reductase genes (arrA), As(V) reductase genes ( $\operatorname{ars} C)$, and As(III) $S$ adenosylmethionine methyltransferase genes (ars $M$ ) in 13 paddy soils collected across Southern China. Sequences grouped with As biotransformation genes are mainly from rice rhizosphere bacteria, such as some Proteobacteria, Gemmatimonadales, and Firmicutes. A significant correlation of gene abundance between ars $C$ and ars $M$ suggests that the two genes coexist well in the microbial As resistance system. Redundancy analysis (RDA)

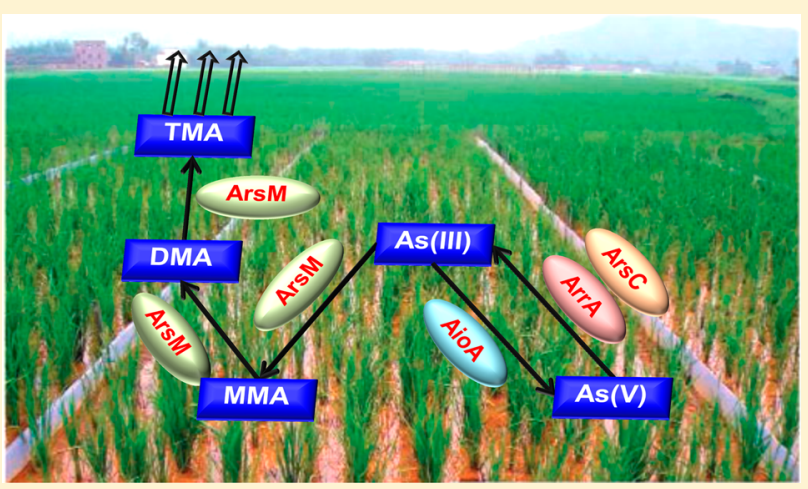
indicated that soil $\mathrm{pH}, \mathrm{EC}$, total $\mathrm{C}, \mathrm{N}, \mathrm{As}$, and $\mathrm{Fe}, \mathrm{C} / \mathrm{N}$ ratio, $\mathrm{SO}_{4}{ }^{2-}-\mathrm{S}, \mathrm{NO}_{3}{ }^{-}-\mathrm{N}$, and $\mathrm{NH}_{4}{ }^{+}-\mathrm{N}$ were the key factors driving diverse microbial community compositions. This study for the first time provides an overall picture of microbial communities involved in As biotransformation in paddy soils, and considering the wide distribution of paddy fields in the world, it also provides insights into the critical role of paddy fields in the As biogeochemical cycle.

\section{INTRODUCTION}

China is the world's largest rice producer, accounting for about $30 \%$ of the total world production (http://beta.irri.org/ statistics), mostly in Southern China. ${ }^{1}$ As a highly toxic metalloid, arsenic (As) contamination in paddy fields has emerged as a serious health concern worldwide, ${ }^{2}$ especially considering that the anaerobic conditions in paddy soils are conducive to As mobilization, ${ }^{3,4}$ resulting in a markedly enhanced bioavailability of As to rice plants. ${ }^{5}$ It is now recognized that consumption of rice constitutes a large proportion of the dietary intake of As for the populations in China and other Asian countries. ${ }^{6-8}$

Microbes are the key drivers for As biotransformation in paddy soils, catalyzing arsenate $(\mathrm{As}(\mathrm{V}))$ reduction, arsenite (As(III)) oxidation and methylation. ${ }^{9}$ Microcosm studies have demonstrated that microbes capable of As reduction, oxidation, and methylation often coexist in paddy soils, and their relative abundance and activity determine the fate of As in the paddy environment and the bioavailability of As to plants. ${ }^{10-12}$ There are two known microbial pathways for $A s(V)$ reduction, the respiratory pathway mediated by arrA genes ${ }^{13}$ and the detoxification pathway mediated by ars $\mathrm{C}$ genes. ${ }^{14} \mathrm{As}(\mathrm{III})$ oxidation is catalyzed by As(III) oxidases, which are encoded by aio $A$ and aio $B$ genes for the two subunits of the enzyme ${ }^{15}$ and have been identified in several heterotrophic and chemoautotrophic microorganisms. ${ }^{16}$ Moreover, As(III) can be methylated by microbes into various organic As species. ${ }^{17}$ Arsenic biomethylation is catalyzed by As(III) S-adenosylmethionine methyltransferase (ArsM), which is encoded by arsM genes. $^{18}$

Received: January 3, 2015

Revised: March 2, 2015

Accepted: March 4, 2015

Published: March 4, 2015 
Although As biotransformation mechanisms have been well studied in microbial pure cultures, ${ }^{19-21}$ the distribution, diversity and abundance of genes responsible for As metabolism in paddy soils have not been well characterized. Metagenomic approach $^{22,23}$ or PCR-based methods ${ }^{24-26}$ have been used to investigate As-related genes in aquatic environments or highly As contaminated environments. In this study, PCR-based methods were used to study aio $A, \operatorname{arr} A$, ars $C$, and $\operatorname{ars} M$ gene abundances and phylogenetic diversities in 13 paddy field samples collected across Southern China. The correlations between different gene abundances were analyzed to investigate the coexistence of As biotransformation genes in paddy soil bacterial genomes. Moreover, in order to obtain a better understanding of how paddy soil properties affect the abundance and diversity of microbial As genes, key factors driving diverse composition of microbial communities involved in As biotransformation in paddy fields were investigated.

\section{MATERIALS AND METHODS}

Soil Sampling. Soil samples were collected from 13 distinct paddy fields across Southern China, Anqing (AQ), Changshu (CS), Jiangmen 1 (JM 1), Jiangmen 2 (JM 2), Fuzhou (FZ), Guilin (GL), Guiyang (GY), Jingzhou (JZ), Jiaxing (JX), Mianyang (MY), Yingtan (YT), Changde (CD), and Zhanjiang (ZJ). The 13 soil samples are from dominant rice production fields located in 11 provinces in China. Soil properties, including $\mathrm{pH}, \mathrm{EC}$, total $\mathrm{C}$, total $\mathrm{N}$, nitrate $\left(\mathrm{NO}_{3}{ }^{-} \mathrm{N}\right)$, ammonium $\left(\mathrm{NH}_{4}^{-}-\mathrm{N}\right)$, total $\mathrm{C} / \mathrm{N}$ ration, total $\mathrm{S}$, sulfate $\left(\mathrm{SO}_{4}{ }^{2-}-\mathrm{S}\right)$, total $\mathrm{Fe}$, total As, and phosphate-extractable As concentrations were determined following standard methods of soil analysis. ${ }^{27-29}$ Details are shown in the Supporting Information.

Nucleic Acid Extraction and PCR. DNA was extracted from $0.5 \mathrm{~g}$ of samples using the FASTDNA SPIN Kit for soil (MP Biomedicals) according to the manufacturer's instructions. PCR amplifications of aio $A$, arrA, ars $C$, and ars $M$ genes were performed with the primers AroAdeg1F/AroAdeg1R and AroAdeg2F/AroAdeg2R, ${ }^{24}$ AS1F/AS1R, ${ }^{25}$ amlt-42-f/amlt-376$\mathrm{r}$ and smrc-42-f/smrc-376-r, ${ }^{26}$ and arsMF1/arsMR2, ${ }^{11}$ respectively. Details are shown in the Supporting Information.

Quantitative PCR of aioA, arrA, ars $C$, and ars $M$ Genes. The abundance of aio $A$, arr $A$, ars $C$, and $\operatorname{ars} M$ genes from 13 samples was estimated using the primers described above by quantitative real-time polymerase chain reaction (qPCR) performed on an iQTM5 Thermocycler (Bio-Rad). Details are shown in the Supporting Information. Assay efficiencies were $95.5 \%$ for aio $A$ genes, $102.6 \%$ for arrA genes, $90.3 \%$ for ars $C$ genes, $100.7 \%$ for ars $M$ genes, and $102.2 \%$ for $16 \mathrm{~S}$ rRNA genes.

Terminal Restriction Fragment Length Polymorphism (T-RFLP) Analysis. PCR amplifications of aioA, arrA, ars $C$, and ars $M$ genes were conducted according to the optimized methods with each of the forward primers labeled with 6carboxyfluorescein (FAM). The labeled PCR products were gel-purified with the Wizard SV Gel and PCR Clean-Up System (Promega) and then digested by the restriction enzyme TaqI (Takara Bio Inc., Japan) at $65{ }^{\circ} \mathrm{C}$ for $4 \mathrm{~h}$. Digestion products were purified and then determined by ABI PRISM 3130XL Genetic Analyzer (Applied Biosystems). Relative abundances of each individual terminal restriction fragments (T-RFs) were calculated based on its peak areas in relation to total peak areas. T-RFs that occurred in at least four replicates and with a percentage $>2 \%$ were listed. These T-RFs were similar to in vivo digestion of the clone sequences in the phylogenetic tree.

Cloning and Community Analysis. According to the results from qPCR and T-RFLP analyses, seven samples (AQ JM 2, FZ, MY, ZJ, GL, and CD) were chosen for cloning and community analysis. They were chosen based on the relative gene abundance and the different microbial community composition ranges which included both samples with the highest gene abundance or biodiversity and the lowest and also included samples with the moderate gene abundance and biodiversity for aio $A, \operatorname{arr} A, \operatorname{ars} C$, and $\operatorname{ars} M$. Details are shown in the Supporting Information. The nucleotide sequences obtained in this study have been submitted into the NCBI GenBank databases under the following accession numbers: aioA genes from KP060099-KP060406, arsC genes from KP060407-KP060549, arsM genes from KP060550KP060962, and arrA genes from KP060963-KP061152.

Statistical Analysis. Spearman's rank-order correlation was used to test the correlations between the relative gene abundances; this nonparametric method was used because the relative gene copy numbers did not follow a normal distribution. Heatmaps and clustering analyses were generated using heatmap tool (heatmap.2) in the gplots package within the statistical program $\mathrm{R}$ 3.1.2. The relative abundances of the OTUs higher than $1 \%$ percentage (the percentage of each OTU to total OTUs) were used to generate heatmaps. Complete linkage clustering of seven samples (AQ JM 2, FZ, $\mathrm{MY}, \mathrm{ZJ}, \mathrm{GL}$, and $\mathrm{CD}$ ) was calculated by the composition and relative abundance of aio $A, \operatorname{arr} A, \operatorname{ars} C$, and $\operatorname{ars} M$ genes. Redundancy analysis (RDA) of environmental variables and the absolute gene copy numbers or T-RFLP based microbial communities were chosen according to the result of detrended correspondence analysis (DCA). Environmental variables were forward selected based on the significance test performed by the Monte Carlo permutation test $(P<0.05)$. These calculations were performed in $\mathrm{R} 3.1 .2$ with the vegan package.

\section{RESULTS}

Soil Properties. Soil properties of the 13 paddy soils are listed in Table S1 in the Supporting Information. Total As concentration varied from 11.7 to $25.4 \mathrm{mg} \mathrm{kg}^{-1}$ (Figure 1). Phosphate-extractable As (0.4-3.6 $\mathrm{mg} \mathrm{kg}^{-1}$ ) represented 3$20 \%$ of the total As, and both inorganic As species (As(III) and As $(\mathrm{V})$ ) and methylated As species (DMA and MMA) were detected in most of the soil samples (Supporting Information, Table S2). As (V) was the dominant As species, accounting for 92-99\% of the phosphate-extractable As concentration, because soils were air-dried before extraction.

Identification and Quantification of the Gene Abundances of As Biotransformation. The aioA, arrA, $\operatorname{ars} C$, and $\operatorname{ars} M$ genes were detected in all samples. To minimize variances caused by different background bacterial abundances, extraction and analytical efficiencies, the absolute gene copy numbers of aioA, arrA, ars $C$, and $\operatorname{ars} M$ (Supporting Information, Figure $S 1$ ) were normalized to that of ambient $16 \mathrm{~S}$ rRNA genes. The relative gene abundances varied for aio $A$ $\left(0.2-6.1 \times 10^{-2}\right), \operatorname{arr} A\left(0.05-8.7 \times 10^{-3}\right)$, ars $C(0.02-1.1 \times$ $\left.10^{-2}\right)$, and $\operatorname{ars} M\left(0.1-2.6 \times 10^{-3}\right)$ (Supporting Information, Figure S2). Among them, aioA genes were the most abundant, while arsM genes were the less abundant. ars $M$ and $\operatorname{ars} C$ showed a strong linear relationship $\left(R^{2}=0.90\right)$ (Figure 2). Spearman's rank-order correlation (rho) analysis also showed that $\operatorname{ars} C$ gene abundances correlated strongly with $\operatorname{ars} M$ (rho = 


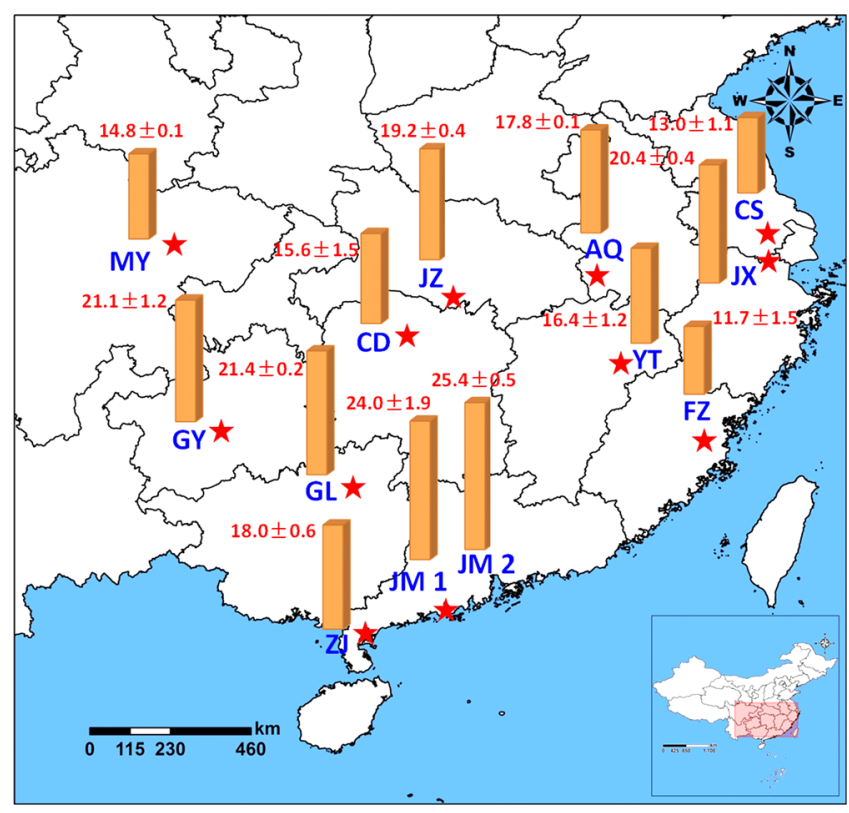

Figure 1. Location and As concentration in 13 paddy soils in Southern China and As concentration in soil samples. The base map used is from the National Fundamental Geographic Information System of China. The orange columns represent As concentration in soil samples.

0.885, $P<0.01)$. The correlations between $\operatorname{arr} A$ and $\operatorname{ars} M$ or $\operatorname{ars} C$ gene abundances were also significant (rho $=0.604$ and $0.714, P<0.05$ and 0.01 , respectively), but these correlations were strongly influenced by the ZJ samples, which appear to be outliers, in fact there was no significant linear relationship between $\operatorname{arr} A$ and ars $M$ or ars $C$ without $\mathrm{ZJ}$ samples $\left(R^{2}=0.15\right.$ and 0.21 , respectively) (Figure 2). The high arrA gene abundances in ZJ samples might be contributed by the microbes represented by T-RFs 34 and $309 \mathrm{bp}$, which were the dominant microbial communities involved in $\mathrm{As}(\mathrm{V})$ respiratory reduction (Supporting Information, Figure S3). There were no significant correlations between aio $A$ genes and the other three genes.

Biodiversity and Community Composition of aioA Genes. T-RFLP analysis showed the variation of microbial community compositions involved in As(III) oxidation in the 13 paddy soils. A total of 10 T-RFs (53, 64, 80, 88, 109, 130, $143,220,347$, and $377 \mathrm{bp}$ ) were detected in aioA gene T-RFLP profiles in soils (Supporting Information, Figure S3). The T-RF 80 bp was dominant in all 13 paddy soils, accounting for 29$75 \%$ of total T-RFs, followed by T-RFs 130, 88, and $220 \mathrm{bp}$. The higher percentage of T-RF $80 \mathrm{bp}$ in JM 2, T-RF $64 \mathrm{bp}$ in MY, T-RF $220 \mathrm{bp}$ in GL, and T-RF $53 \mathrm{bp}$ in YT and CD contributed to their different microbial communities from the other samples, which was also shown in RDA based on aioA TRFLP profiles (Figure 3). Heatmap of aioA genes showed that the microbes responsible for As(III) oxidation represented by OTU 1 and 2 were the dominant genera in the 7 selected paddy soils. Cluster analysis based on aio $A$ gene sequences also showed that sample JM 2 separated from FZ, AQ and ZJ, and CD separated from MY and GL (Figure 4). Phylogenetic analysis of the 646 aioA sequences from the seven selected samples allowed the identification of 308 unique OTUs based on a $97 \%$ cutoff (Supporting Information, Figure S5). The microbes were mainly $\alpha$-Proteobacteria $(14 \%)$ and $\beta$-Proteobacteria (37\%), including Rhizobiales and Burkholderiales, together with three undefined clusters along with amplified aioA genes from rice rhizosphere, rice root, aquatic sediment, and As contaminated soil. ${ }^{10,24,30}$
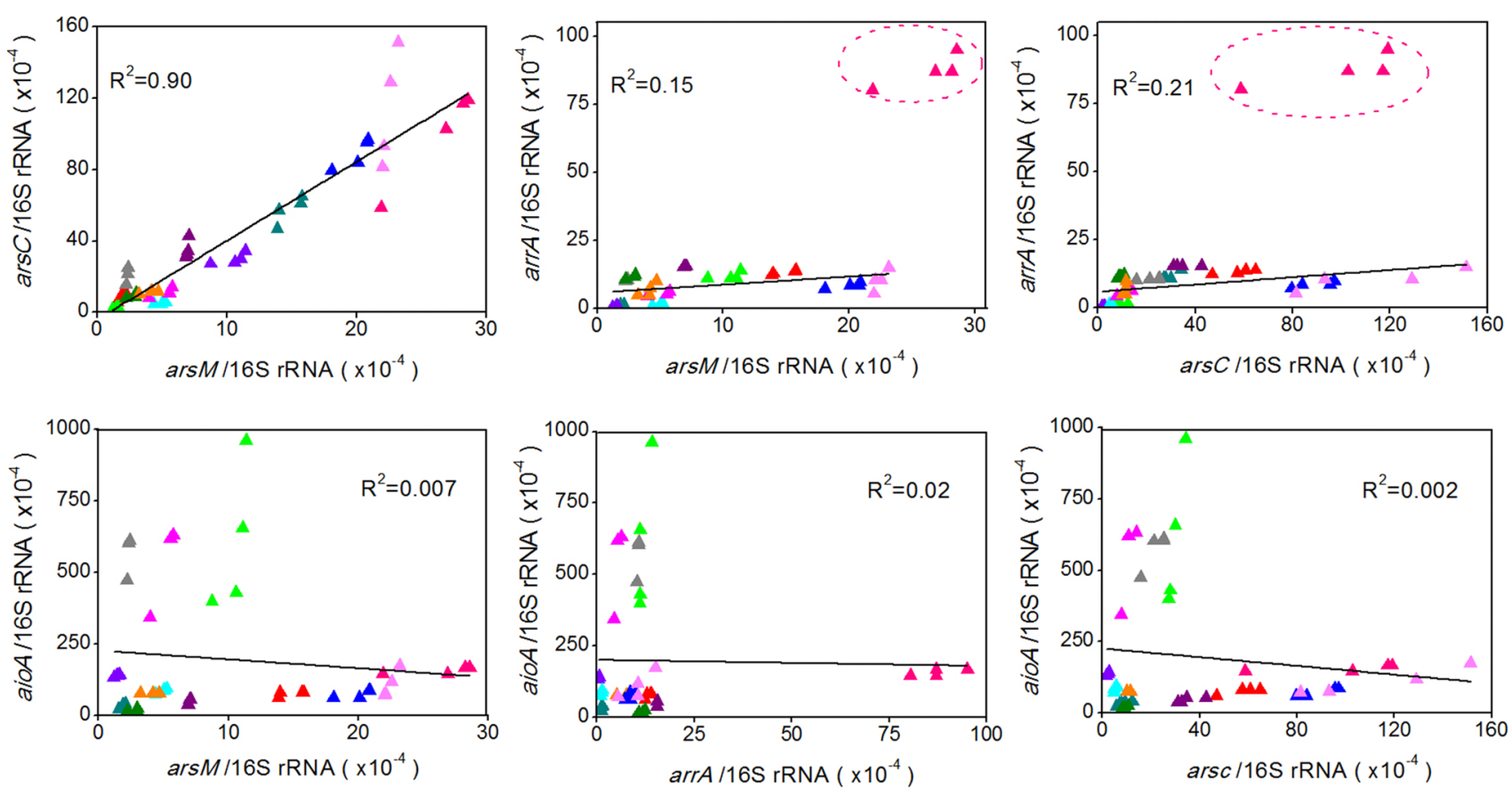

Figure 2. Relationships of relative gene abundances of aioA, arrA, ars $C$, and $\operatorname{ars} M$ from the 13 paddy soils in Southern China. Lines are fitted according to linear fitting in OriginPro 8 program. To minimize variances caused by different background bacterial abundances, extraction and analytical efficiencies, the absolute gene copy numbers of aioA, $\operatorname{arrA}, \operatorname{ars} C$, and $\operatorname{ars} M$ (Supporting Information, Figure S1) were normalized to that of ambient $16 \mathrm{~S}$ rRNA genes. For the relationships between $\operatorname{arr} A$ and $\operatorname{ars} M$ or $\operatorname{ars} C$ gene abundances, $\mathrm{ZJ}$ samples were shown as outliers in the dashed circle. 

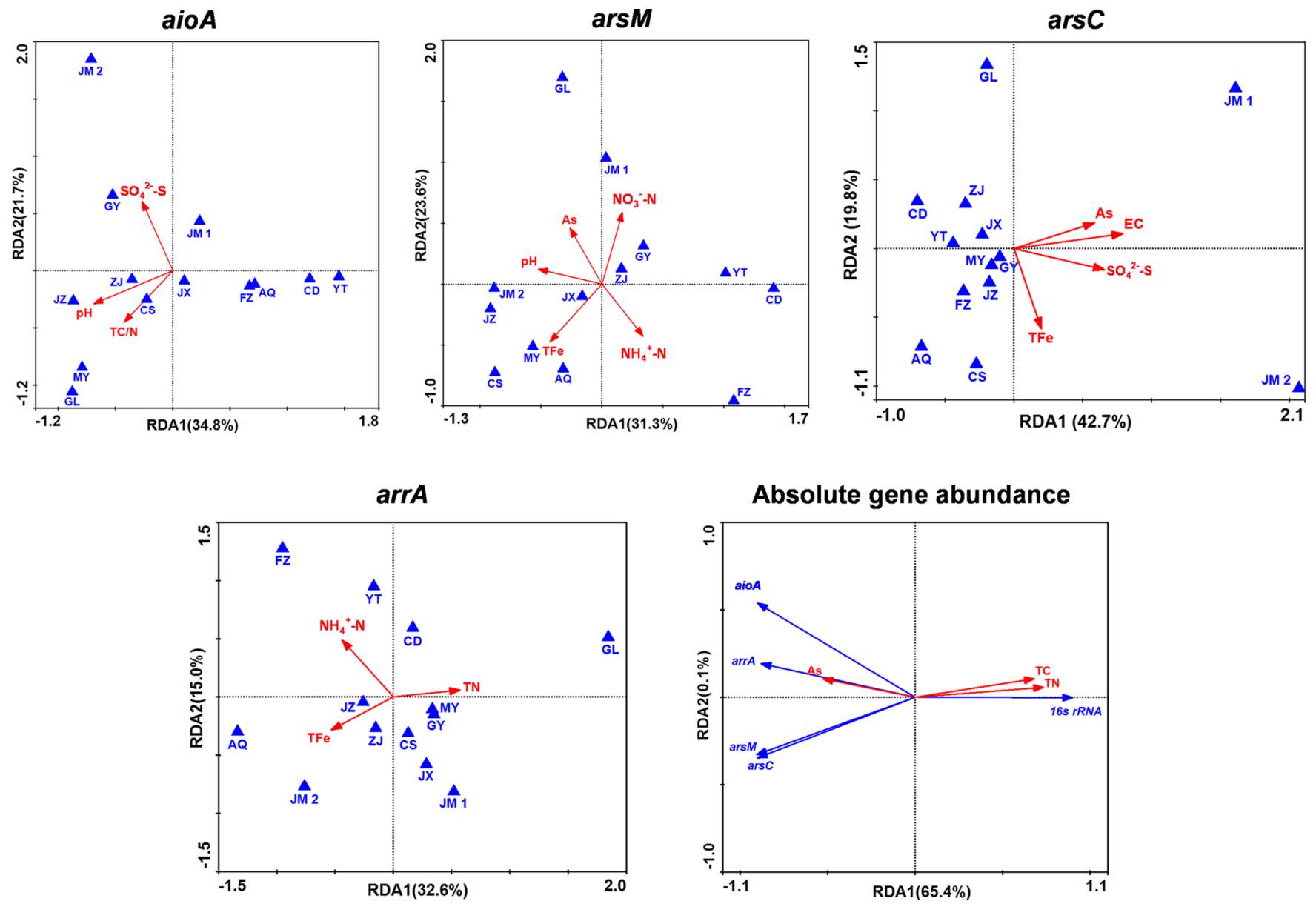

Figure 3. RDA correlation biplot of aioA, arrA, arsC, and $\operatorname{ars} M$ microbial communities based on T-RFLP profiles with environmental factors and absolute gene abundances of aioA, arrA, ars $C$, ars $M$, and $16 \mathrm{~S}$ rRNA with environmental factors. Arrows indicate the direction and magnitude of each environmental factor associated with bacterial communities and gene abundance.

Biodiversity and Community Composition of arrA Genes. Seven T-RFs $(34,58,117,123,192,309$, and 379 bp) were detected in arrA gene T-RFLP profiles (Supporting Information, Figure S3). T-RF 34 bp had the highest relative abundance $(42-91 \%)$ in all the 13 paddy soils, followed by $\mathrm{T}$ RFs 58 and 123 bp except the AQ and FZ samples, for which the dominant T-RFs were 123 (49\%) and 379 bp (52\%), respectively. In sample GL, the percentage of T-RF $34 \mathrm{bp}$ was the highest, which contributed most to its difference from the other samples. The differences of microbial community compositions for sample AQ FZ, and GL were also shown in RDA based on arrA T-RFLP profiles (Figure 3). Heatmap of $\operatorname{arr} A$ genes revealed that the microbes associated with respiratory $\mathrm{As}(\mathrm{V})$ reduction represented by OTU 1-5 were the dominant genera in the 7 selected paddy soils. Cluster analysis based on arrA sequences showed that sample AQ was the most different from the other paddy soils (Figure 4). The phylogenetic tree of arrA sequences was constructed with 190 defined OTUs (identity >97\%) after analysis of 613 arrA clone sequences (Supporting Information, Figure S6). The arrA sequences could be grouped into seven clusters, and most of the sequences were aligned to the arr $A$ genes of uncultured bacteria identified in paddy soil, sediments, and bioreactors. ${ }^{10,25,31-33}$ Some of the arrA sequences were related to Geobacter uraniireducens.

Biodiversity and Community Composition of arsC Genes. Five T-RFs (38, 53, 92, 146, and 203 bp) were detected in ars $C$ gene T-RFLP profiles (Supporting Information, Figure S3). T-RFs of 38, 53, and 146 bp were detected in all the 13 paddy fields, and $53 \mathrm{bp}$ was the dominant T-RF, accounting for 37-66\% of the total T-RFs. T-RF 203 and $92 \mathrm{bp}$ were detected only in samples CS, GL, JZ and AQ, YT, CD, respectively. The higher percentage of T-RF $146 \mathrm{bp}$ in samples JM 1, JM 2, and GL contributed to their distinct microbial community compositions to the other samples and coincided with the result of RDA based on arsC T-RFLP profiles (Figure 3). Heatmap of $\operatorname{ars} C$ genes showed the dominant genera involved in $\mathrm{As}(\mathrm{V})$ reduction in these paddy soils, and cluster analysis based on $\operatorname{ars} C$ sequences also revealed that samples GL and JM 2 separately from the other paddy soils (Figure 4). The phylogenetic tree of ars $C$ sequences was constructed with 143 defined OTUs (identity >97\%) after analysis of 615 arsC clone sequences (Supporting Information, Figure S7) and revealed a dominance of bacteria belonging to the Proteobacteria phylum $(\alpha$-Proteobacteria and $\gamma$-Proteobacteria with $43 \%$ and $8 \%$, respectively), including Hoeflea, Sinorhizobium, Mesorhizobium, Polymorphum, and Enterobacter, and also three unknown clusters, mainly microbes associated with the rice rhizosphere, rice root, and As contaminated soils. ${ }^{10,14,26}$

Biodiversity and Community Composition of ars $M$ Genes. According to the T-RFLP analysis of arsM genes, $10 \mathrm{~T}$ RFs (32, 59, 70, 83, 137, 158, 203, 224, 268, and 312 bp) were detected in the soils (Supporting Information, Figure S3). T-RF 83 bp was dominant in samples AQ CS, JM 2, JZ, JX, MY, and 

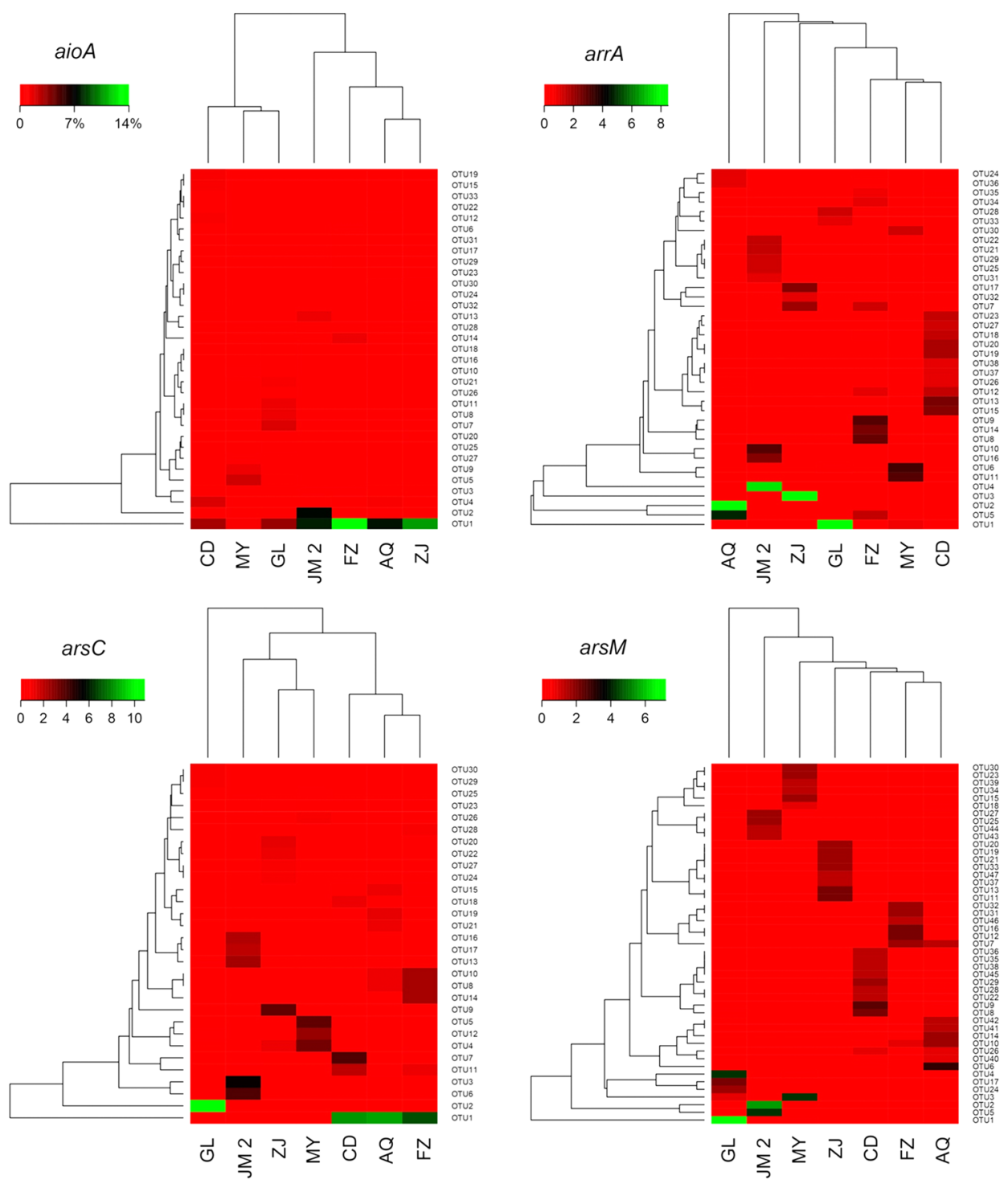

Figure 4. Abundance and diversity of microbial aioA, $\operatorname{arrA}$, arsC, and arsM genes in seven paddy soils (AQ, JM 2, FZ, MY, ZJ, GL, and CD) based on OTU. Complete linkage clustering of seven samples was calculated by the composition and relative abundance of aioA, $\operatorname{arr} A$, ars $C$, and $\operatorname{ars} M$ genes. OTUs that have higher than $1 \%$ percentage (the percentage of each OTU to total OTUs) were shown in the heat map. Scales of green, black, and red indicated the relative abundance of each OTU in different samples.

ZJ, with the relative abundances ranging from $34 \%$ to $46 \%$. In the other six soils (JM 1, FZ, GL, GY, YT, and CD), T-RF 59 bp showed the highest abundance, accounting for 34-60\%. The appearance of T-RF $70 \mathrm{bp}$ in samples GL, JM 1, and GY and the highest abundance of T-RF $59 \mathrm{bp}$ in samples FZ, YT, and $\mathrm{CD}$ contributed to their different microbial community compositions from the other samples, which were also revealed by RDA based on arsM T-RFLP profiles (Figure 3). The dominant genera involved in As methylation in these paddy soils were revealed in heatmap of ars $M$ genes, and samples GL and JM 2 were shown as the most distinct from the other paddy soils in cluster analysis based on ars $M$ sequences (Figure 4). Analysis of the 656 arsM sequences from the seven selected samples allowed the identification of 413 unique OTUs based on a $97 \%$ cutoff (Supporting Information, Figure S8). These sequences belonged to Gemmatimonadales (16\%), Firmicutes (9\%), Actinobacteria (11\%), $\alpha$-Proteobacteria (22\%), $\beta$-Proteobacteria (6\%), $\delta$-Proteobacteria (6\%), and Archaea (6\%) and to a lesser extent to two unknown clusters containing sequences from rice rhizosphere soil and rice root. ${ }^{11}$

RDA of Environmental Factors and As Biotransformation Microbial Community Compositions and Gene Abundances. According to RDA (Figure 3), the environmental factors $\mathrm{pH}(P=0.001), \mathrm{SO}_{4}{ }^{2-}-\mathrm{S}(P=0.02)$, and total $\mathrm{C} / \mathrm{N}$ ratio $(P=0.02)$ significantly explained the variation in the microbial community compositions based on aio $A$ genes, and the first and second axis accounted for $34.8 \%$ and $21.7 \%$ of the total variance, respectively. The $\mathrm{pH}$ value explained the most variation $(32.1 \%)$ of microbial community compositions involved in $\mathrm{As}$ (III) oxidation, followed by $\mathrm{SO}_{4}{ }^{2-}-\mathrm{S}$ (21.8\%) and total $\mathrm{C} / \mathrm{N}$ ratio $(21.1 \%)$. For $\operatorname{ars} M$ based microbial communities, total Fe $(P=0.02), \mathrm{NO}_{3}^{-}-\mathrm{N}(P=0.02), \mathrm{pH}(P=$ $0.04)$, total As $(P=0.04)$, and $\mathrm{NH}_{4}{ }^{+}-\mathrm{N}(P=0.03)$ significantly contributed to the variant compositions of microbial communities, and the first and second axis accounted for $31.3 \%$ and $23.6 \%$ of the total variance, respectively. Total $\mathrm{Fe}$ explained the most variation $(22.9 \%)$ and followed by $\mathrm{NO}_{3}{ }^{-}-\mathrm{N}$ 
(19.7\%), $\mathrm{pH}(19.4 \%)$, total As (18.6\%), and $\mathrm{NH}_{4}{ }^{+}-\mathrm{N}(16.3 \%)$. For ars $C$ based microbial communities, EC $(P=0.002), \mathrm{SO}_{4}{ }^{2-}$ $\mathrm{S}(P=0.03)$, total As $(P=0.03)$ and $\mathrm{Fe}(P=0.05)$ explained a significant amount of the variability in microbial community compositions, and the first and second axis accounted for $42.7 \%$ and $19.8 \%$ of the total variance, respectively. EC explained the most $(41.4 \%)$ variation and followed by $\mathrm{SO}_{4}{ }^{2-}-\mathrm{S}(29.3 \%)$, total As $(24.1 \%)$, and $\mathrm{Fe}(17.8 \%)$. For microbial communities based on arrA genes, the environmental factors $\mathrm{NH}_{4}{ }^{+}-\mathrm{N}(P=0.01)$, total Fe $(P=0.03)$, and $\mathrm{N}(P=0.04)$ significantly correlated with the variation of microbial community compositions, and the first and second axis accounted for $32.6 \%$ and $15.0 \%$ of the total variance, respectively. Among them, $\mathrm{NH}_{4}{ }^{+}-\mathrm{N}$ explained the most variation of microbial community compositions associated with respiratory $\mathrm{As}(\mathrm{V})$ reduction (20.4\%) and followed by total $\mathrm{Fe}(20.3 \%)$ and $\mathrm{N}(20.3 \%)$. For the absolute gene abundances of aioA, arrA, arsC, ars $M$, and $16 \mathrm{~S}$ rRNA, total $\mathrm{N}(P=0.02), \mathrm{C}(P=0.02)$, and As $(P=0.03)$ significantly explained the variance in 13 paddy soils, and the first and second axis accounted for $65.4 \%$ and $0.1 \%$ of the total variance separately. Among them, total $\mathrm{N}$ explained the most variation (43.3\%) and was followed by C (37.4\%) and As (22.5\%).

\section{DISCUSSION}

High Abundance of aioA Genes in Paddy Soils. The abundance of aio $A$ genes was almost 10 times higher than the other genes (Supporting Information, Figure S2). A higher diversity of aio $A$ compared with $\operatorname{arr} A$ and $\operatorname{ars} C$ genes was also revealed by Shannon and Simpson diversity indices (Supporting Information, Table S4), indicating a high potential for microbial As oxidation in paddy soils. The major groups of As oxidizing bacteria, i.e., the Rhizobiales and Burkholderials in $\alpha$ Proteobacteria and $\beta$-Proteobacteria (Supporting Information, Figure S5), were typically rhizospheric microbes. ${ }^{10}$ These microbes have been shown to contribute significantly to the oxidation of As(III) in soils under both aerobic and anaerobic conditions. ${ }^{34}$ Previous studies have shown that the abundance of As(III)-oxidizing microbes could enhance As(III) oxidation to $\mathrm{As}(\mathrm{V})$ in the rice rhizosphere, resulting in decreased As mobility and bioavailability because $\mathrm{As}(\mathrm{V})$ can be sequestered on $\mathrm{Fe} / \mathrm{Mn}$ hydroxide/oxyhydroxide in rhizosphere soil and on rice roots. ${ }^{35,36}$ For microbes, As(III) oxidation is also one of the As detoxification mechanisms, because the product of oxidation, $\mathrm{As}(\mathrm{V})$, is less toxic than $\mathrm{As}(\mathrm{III}) .{ }^{15}$ In addition, compared with most of the other samples, the lower aio $A$ gene abundances in samples ZJ, AQ, CD, and FZ, which had been drained to different extent at sampling, indicated that the decrease of mobile As(III) under nonflodded period ${ }^{3}$ resulted in the reduction of aio $A$ gene abundances. ${ }^{10}$

Both arrA and arsC Genes Were Identified in Paddy Soils. Genes involved in both respiratory $\mathrm{As}(\mathrm{V})$ reduction and detoxifying $\mathrm{As}(\mathrm{V})$ reduction were identified in the paddy soils. The abundance of $\operatorname{arr} A$ genes was much lower than that of $\operatorname{ars} C$ (Supporting Information, Figure S2), possibly because arrA genes present only in anaerobic microbes, ${ }^{37}$ which restricts its abundance in the paddy soils. Under flooded conditions, because of reductive dissolution of the $\mathrm{Fe}$ (hydr)oxide and reduction of $\mathrm{As}(\mathrm{V})$ to $\mathrm{As}(\mathrm{III})$, arsenic is quickly released from the soil to the pore water. ${ }^{3}$ The $\mathrm{As}(\mathrm{V})$ respiring bacteria could reduce $\mathrm{As}(\mathrm{V})$ under the anaerobic conditions to gain energy. ${ }^{13,38}$ arrA sequences closely related to Geobacter uraniireducens species have been identified in this study, which has also been confirmed by Héry et al. that reported the arrA amplified from West Bengali sediments showed high amino acid sequence identity to sequences of putative arr $A$ genes in genomes of Geobacter uraniireducens and G. lovleyi. ${ }^{39}$ Moreover, arrA related to Geobacter species have been frequently detected in As rich sediments. ${ }^{40}$ Unlike $\operatorname{arr} A$, the detoxification reductase genes $\operatorname{ars} C$ is present in both aerobic and anaerobic microbes ${ }^{19,37}$ and has been identified in environmental samples and rice rhizosphere. ${ }^{11,30}$ In this study, the microbes responsible for detoxification As(V) reduction belonged to some typically rhizospheric microbes, such as Rhizobiales and Pseudomonadales (Supporting Information, Figure S7) and could contribute to $\mathrm{As}(\mathrm{V})$ reduction and mobility in paddy soils under both aerobic and anaerobic conditions.

High Diversity of arsM Genes in Paddy Soils. Although ars $M$ gene abundance was the lowest when compared with the other genes (Supporting Information, Figure S2), the diversity (estimated by Shannon and Simpson diversity indices) and the OTU richness (estimated by Chao and ACE) of arsM genes were much higher than aioA, arr A, and ars $C$ genes at a $97 / 90 \%$ similarity cutoff in almost all the samples (Supporting Information, Table S4), suggesting a highly diverse microbial communities involved in As methylation in paddy fields. These diverse microbes could contribute to the presence of monomethylarsenate (MMAs(V)) and dimethylarsenate (DMAs $(\mathrm{V})$ ) detected in the soils (Supporting Information, Table S2). The representative arsM-containing genera in Proteobacteria, Gemmatimonadales, and Firmicutes (Supporting Information, Figure S8) have been identified as the major microbial community in the rice rhizosphere, ${ }^{41}$ and their significant contribution to As methylation has also been reported. $^{11}$ Various volatile As species (mono-, di-, and trimethylarsines) can be generated during the As methylation process $^{42}$ and have been detected in the atmosphere above paddy fields. ${ }^{43}$ The wide distribution of As(III) methyltransferases in the 13 paddy soils underlines the potential for As methylation resulting in not only the accumulation of methylated As species in rice ${ }^{44}$ but also volatilization of As into the atmosphere. Previous studies have estimated that natural origin represented $62 \%$ of the volatile As released to the atmosphere, ${ }^{45}$ and As biovolatilization accounted for $58 \%$ of natural As emissions. ${ }^{46}$ Mestrot et al. ${ }^{43}$ reported that As emitted from Bangladesh and Spanish paddy soils to the atmosphere was 23-51 and 4.5-9.2 t/year, respectively, and demonstrated the sizable contribution of paddy fields to As biovolatilization.

Strong Correlation of arsC and arsM Gene Abundance. It is interesting to note that $\operatorname{ars} C$ gene abundance correlated strongly with ars $M$ in the 13 paddy soils (Figure 2). ars $C$ gene encodes the cytoplasmic ArsC reductase and catalyzes the cellular reduction of $\mathrm{As}(\mathrm{V})$ to $\mathrm{As}(\mathrm{III}){ }^{19}$ The toxic As(III) can either be subsequently extruded by the efflux system $^{37}$ or methylated to less toxic methylated As species (MMAs(V), DMAs(V), and volatile TMAs(III)) by ArsM and thus to complete the detoxification processes. ${ }^{20}$ Studies of microbes have shown that ars $M$ gene is often in the gene cluster adjacent to other genes encoding As-resistance proteins. For example, Rhodopseudomonas palustris CGA009 or Synechocystis sp. PCC6803 both contain genes encoding ArsM, arsenical compound resistance 3 (ACR3), and ArsC for As resistance. $^{18,21}$ Therefore, the strong correlation between $\operatorname{ars} C$ and ars $M$ gene abundances strongly implies that ars $M$ gene coexists well with the ars $C$ gene in the microbial As resistance system, especially for microbes in paddy fields. Moreover, there was an 
indication of a similar clustering of soils from the 7 different geographical regions for microbes involved in $\mathrm{As}(\mathrm{V})$ reduction and As methylation based on ars $C$ and ars $M$ gene diversities (Figure 4). This result suggests that similar compositions of microbial communities are involved in $\mathrm{As}(\mathrm{V})$ reduction and As methylation in paddy soils.

Soil Properties Explaining Variations in Gene Abundances and Microbial Community Compositions Involved in As Biotransformation. Among the soil properties tested (Figure 3), total As concentration was found to be positively correlated with the abundances of aio $A, \operatorname{arr} A, \operatorname{ars} C$, and ars $M$ genes. In contrast, $16 \mathrm{~S}$ rRNA gene abundance had a negative correlation with As concentration but a positive correlation with total $\mathrm{C}$ and $\mathrm{N}$ concentrations, implying that $\mathrm{As}$ in the paddy fields possibly exerted a selection pressure on soil microbes resulting in decreased bacterial abundance. However, the presence of As in soils might result in higher abundance of microbes involved in As biotransformation, as Lami et al. reported that when amended with $200 \mu \mathrm{mol} \mathrm{kg}{ }^{-1}$ As(III), the total number of bacterial taxa decreased by 2 -fold but some of the As(III) oxidizing bacterial groups increased up to 20 -fold. ${ }^{47}$ Soil $\mathrm{pH}$ had a significant effect on the composition of microbial communities associated with both As(III) oxidation and As methylation, probably due to the narrow $\mathrm{pH}$ ranges for optimal growth of bacteria. ${ }^{48}$ The concentration of $\mathrm{SO}_{4}{ }^{2-}-\mathrm{S}$ also explained $21.8 \%$ and $29.3 \%$ variation in the structure of microbial communities responsible for As(III) oxidation and detoxification $\mathrm{As}(\mathrm{V})$ reduction, respectively. A previous study with metaproteomic method showed the role of several dominant bacteria in highly As contaminated acid mine drainage recycling of both mineral and organic resources, such as As, Fe, S, urea, vitamins, nucleosides, and amino acids. ${ }^{27}$ In this case, microbes responsible for As(III) oxidation and $\mathrm{As}(\mathrm{V})$ reduction might share some microbial groups with that capable of sulfur oxidation, and it is reasonable to expect that $\mathrm{SO}_{4}{ }^{2-}-\mathrm{S}$ concentration may have a significant influence on these microbial community composition. Total Fe concentration was another significant environmental factor responsible for the variation in the composition of microbial communities involved in respiratory $\mathrm{As}(\mathrm{V})$ reduction, detoxification $\mathrm{As}(\mathrm{V})$ reduction and As methylation in paddy soils. Many studies have demonstrated that iron oxyhydroxides could sequester As $(\mathrm{V})$, and the reduction of iron oxyhydroxides could increase As bioavailability in paddy soils. ${ }^{49,50}$ Therefore, it could be Fe oxides that contribute to microbial community diversity. For microbes with ars $C$ and $\operatorname{ars} M$ genes, the concentrations of both $\mathrm{Fe}$ and As were the dominant environmental factors driving the variation in bacterial community composition. While the concentration of total $\mathrm{N}, \mathrm{NH}_{4}{ }^{+}-\mathrm{N}$, and total $\mathrm{Fe}$ were the dominant environmental factors responsible for diverse compositions of microbial communities involved in respiratory As $(\mathrm{V})$ reduction. This could be explained as a previous study showed microbially mediated redox processes in paddy soils are strongly related to the microbial accessibility of $\mathrm{C}, \mathrm{N}$, and $\mathrm{Fe}{ }^{1}$ Moreover, the concentrations of $\mathrm{NH}_{4}{ }^{-}-\mathrm{N}$ and $\mathrm{NO}_{3}^{-}-\mathrm{N}$ were also potential drivers of microbial community compositions involved in As methylation as shown in RDA in this study. In addition, the EC value was another significant environmental factor affecting microbial community structures, as it contributed most to the variation in the composition of microbial communities involved in $\mathrm{As}(\mathrm{V})$ reduction. It is possible that the effect of EC is related to its significant correlation with As concentration in the 13 soils ( $\mathrm{rho}=0.709, P$ $<0.01$, Supporting Information, Table S5).

Implications. This study shows the ubiquitous distribution of microbes associated with As redox changes and methylation in paddy soils. These microbes are likely to play a key role in driving the biogeochemical cycle of As in the paddy environment and affecting As accumulation by rice plants. ${ }^{10-12}$ The distribution patterns of the dominant genera involved in As biotransformation and the cluster analysis based on aio $A$, $\operatorname{arr} A, \operatorname{ars} C$, and $\operatorname{ars} M$ diversities suggest a clustering of soils from different geographical regions, i.e., the separation of southeast samples (AQ FZ, JM 2, and ZJ) from the southwest samples (MY, CD, and GL) based on aio $A$ and $\operatorname{arr} A$ genes, and the separation of samples GL, ZJ, and JM 2 which locate at the most southern part in China from the other samples based on ars $C$ and ars $M$ genes (Figure 1), implying the different microbe-mediated As biotransformation abilities in variant paddy soils based on geographic region and might result in different As species and concentrations accumulated by rice plants. The separations of different geographical regions might result from different soil properties as revealed by $\mathrm{RDA}$ analysis based on aioA, arrA, ars $C$, and ars $M$ T-RFLP profiles, which indicated the similar separation patterns for these samples (Figure 3). For aioA and arrA genes, the lower soil $\mathrm{pH}$ value and total $\mathrm{C} / \mathrm{N}$ ratio in samples $\mathrm{MY}$ and GL, and the high concentration of total $\mathrm{N}$ in samples MY, GL, and CD contributed the most to the separation patterns. While for ars $C$ and ars $M$ genes, the different separation patterns might be due to the higher As concentrations in samples GL, ZJ, and JM 2. The environmental factors, such as $\mathrm{pH}$, total $\mathrm{C}, \mathrm{N}$, and $\mathrm{Fe}$, appear to drive the variation in gene abundance and microbial community associated with As biotransformation. These factors have also been implicated in the As biogeochemical cycle in paddy soils. ${ }^{1,4}$ Manipulating these environmental factors through cultivating rice aerobically or minimizing organic matter amendments in paddy soil can effectively reduce As accumulation in rice grain. ${ }^{5,49}$ Given that paddy soils make up the largest anthropogenic wetlands on earth, which are highly modified by anthropogenic activities, ${ }^{1}$ the identified phylogenetic and ecological diversity of microbes involved in As redox changes and methylation in paddy soils underlines their critical role in the global As biogeochemical cycle. Future in situ studies should be carried out to investigate how paddy conditions impact on As biotransformation gene abundance and microbial community composition by comparing adjacent paddy and nonpaddy soils.

\section{ASSOCIATED CONTENT}

\section{S Supporting Information}

Gene abundances; community structure of $\operatorname{aio} A, \operatorname{arr} A$, $\operatorname{ars} C$, and ars $M$ genes; rarefaction curve of clone libraries; phylogeny of aio $A$, $\operatorname{arr} A, \operatorname{ars} C$, and $\operatorname{ars} M$ sequences; soil location and properties; primers sequences and PCR thermal cycling parameters; diversity and richness estimators (Shannon, Simpson, ACE and Chao1); Spearman's rank-order correlation of soil properties. This material is available free of charge via the Internet at http://pubs.acs.org.

\section{AUTHOR INFORMATION}

\section{Corresponding Author}

*Phone: 86-592-6190997; fax: 86-592-6190997; e-mail: ygzhu@rcees.ac.cn. 


\section{Notes}

The authors declare no competing financial interest.

\section{ACKNOWLEDGMENTS}

This study is financially supported by Natural Science Foundation of China (Grant No. 41330853). We would like to thank Shu-Wei Wang in the Institute of Soil Science, Chinese Academy of Science for helping collecting paddy soil samples. We also thank Xin- $\mathrm{Li} \mathrm{An}$ and Hui-Juan $\mathrm{Xu}$ in the Institute of Urban Environment, Chinese Academy of Sciences for helping with the DNA extraction and providing the National Fundamental Geographic Information System of China.

\section{REFERENCES}

(1) Koegel-Knabner, I.; Amelung, W.; Cao, Z.; Fiedler, S.; Frenzel, P.; Jahn, R.; Kalbitz, K.; Koelbl, A.; Schloter, M. Biogeochemistry of paddy soils. Geoderma 2010, 157 (1-2), 1-14.

(2) Mandal, B. K.; Suzuki, K. T. Arsenic round the world: a review. Talanta 2002, 58 (1), 201-235.

(3) Takahashi, Y.; Minamikawa, R.; Hattori, K. H.; Kurishima, K.; Kihou, N.; Yuita, K. Arsenic behavior in paddy fields during the cycle of flooded and non-flooded periods. Environ. Sci. Technol. 2004, 38 (4), 1038-1044.

(4) Yamaguchi, N.; Nakamura, T.; Dong, D.; Takahashi, Y.; Amachi, S.; Makino, T. Arsenic release from flooded paddy soils is influenced by speciation, Eh, $\mathrm{pH}$, and iron dissolution. Chemosphere 2011, 83 (7), 925-932.

(5) Xu, X. Y.; McGrath, S.; Meharg, A.; Zhao, F. J. Growing rice aerobically markedly decreases arsenic accumulation. Environ. Sci. Technol. 2008, 42 (15), 5574-5579.

(6) Heikens, A. Arsenic contamination of irrigation water, soil and crops in Bangladesh: Risk implications for sustainable agriculture and food safety in Asia. In FAO Corporate Document Repository; FAO Regional Office for Asia and the Pacific: Bangkok, Thailand, 2006.

(7) Li, G.; Sun, G. X.; Williams, P. N.; Nunes, L.; Zhu, Y. G. Inorganic arsenic in Chinese food and its cancer risk. Environ. Int. 2011, 37 (7), 1219-1225.

(8) Mondal, D.; Polya, D. A. Rice is a major exposure route for arsenic in Chakdaha block, Nadia district, West Bengal, India: A probabilistic risk assessment. Appl. Geochem. 2008, 23 (11), 29872998.

(9) Meharg, A. A.; Zhao, F. J. Biogeochemistry of arsenic in paddy environments. In Arsenic \& Rice; Springer Science \& Business Media Press: Dordrecht, The Netherlands, 2012; pp 71-101.

(10) Jia, Y.; Huang, H.; Chen, Z.; Zhu, Y. G. Arsenic uptake by rice is influenced by microbe-mediated arsenic redox changes in the rhizosphere. Environ. Sci. Technol. 2014, 48 (2), 1001-1007.

(11) Jia, Y.; Huang, H.; Zhong, M.; Wang, F. H.; Zhang, L. M.; Zhu, Y. G. Microbial arsenic methylation in soil and rice rhizosphere. Environ. Sci. Technol. 2013, 47 (7), 3141-3148.

(12) Zhao, F. J.; Harris, E.; Yan, J.; Ma, J.; Wu, L.; Liu, W. J.; McGrath, S. P.; Zhou, J. Z.; Zhu, Y. G. Arsenic methylation in soils and its relationship with microbial arsM abundance and diversity, and As speciation in rice. Environ. Sci. Technol. 2013, 47 (13), 7147-7154.

(13) Malasarn, D.; Saltikov, W.; Campbell, K. M.; Santini, J. M.; Hering, J. G.; Newman, D. K. arrA is a reliable marker for As(V) respiration. Science 2004, 306 (5695), 455-455.

(14) Villegas-Torres, M. F.; Bedoya-Reina, O. C.; Salazar, C.; VivesFlorez, M. J.; Dussan, J. Horizontal ars $C$ gene transfer among microorganisms isolated from arsenic polluted soil. Int. Biodeter. Biodegr. 2011, 65 (1), 147-152.

(15) Slyemi, D.; Bonnefoy, V. How prokaryotes deal with arsenic. Environ. Microbiol. Rep. 2012, 4 (6), 571-586.

(16) Hamamura, N.; Macur, R. E.; Korf, S.; Ackerman, G.; Taylor, W. P.; Kozubal, M.; Reysenbach, A. L.; Inskeep, W. P. Linking microbial oxidation of arsenic with detection and phylogenetic analysis of arsenite oxidase genes in diverse geothermal environments. Environ. Microbiol. 2009, 11 (2), 421-431.

(17) Zhu, Y. G.; Yoshinaga, M.; Zhao, F. J.; Rosen, B. P. Earth abides arsenic biotransformations. Annu. Rev. Earth Planet. Sci. 2014, 42, $443-467$.

(18) Qin, J.; Rosen, B. P.; Zhang, Y.; Wang, G. J.; Franke, S.; Rensing, C. Arsenic detoxification and evolution of trimethylarsine gas by a microbial arsenite $S$-adenosylmethionine methyltransferase. Proc. Natl. Acad. Sci. U.S.A. 2006, 103 (7), 2075-2080.

(19) Silver, S.; Phung, L. T. Genes and enzymes involved in bacterial oxidation and reduction of inorganic arsenic. Appl. Environ. Microbiol. 2005, 71 (2), 599-608.

(20) Ye, J.; Rensing, C.; Rosen, B. P.; Zhu, Y. G. Arsenic biomethylation by photosynthetic organisms. Trends. Plant Sci. 2012, 17 (3), 155-162.

(21) Zhang, S. Y.; Rensing, C.; Zhu, Y. G. Cyanobacteria-mediated arsenic redox dynamics is regulated by phosphate in aquatic environments. Environ. Sci. Technol. 2013, 48 (2), 994-1000.

(22) Cai, L.; Yu, K.; Yang, Y.; Chen, B. W.; Li, X. D.; Zhang, T. Metagenomic exploration reveals high levels of microbial arsenic metabolism genes in activated sludge and coastal sediments. Appl. Microbiol. Biotechnol. 2013, 97 (21), 9579-9588.

(23) Luo, J. M.; Bai, Y. H.; Liang, J. S.; Qu, J. H. Metagenomic approach reveals variation of microbes with arsenic and antimony metabolism genes from highly contaminated soil. PLoS One 2014, 9 (10), e108185.

(24) Inskeep, W. P.; Macur, R. E.; Hamamura, N.; Warelow, T. P.; Ward, S. A.; Santini, J. M. Detection, diversity and expression of aerobic bacterial arsenite oxidase genes. Environ. Microbiol. 2007, 9 (4), 934-943.

(25) Song, B.; Chyun, E.; Jaffé, P. R.; Ward, B. B. Molecular methods to detect and monitor dissimilatory arsenate-respiring bacteria (DARB) in sediments. FEMS Microbiol. Ecol. 2009, 68 (1), 108-117.

(26) Sun, Y. M.; Polishchuk, E. A.; Radoja, U.; Cullen, W. R. Identification and quantification of ars $C$ genes in environmental samples by using real-time PCR. J. Microbiol. Methods 2004, 58 (3), $335-349$.

(27) Bertin, P. N.; Heinrich-Salmeron, A.; Pelletier, E.; GoulhenChollet, F.; Arsène-Ploetze, F.; Gallien, S.; Lauga, B.; Casiot, C.; Calteau, A.; Vallenet, D. Metabolic diversity among main microorganisms inside an arsenic-rich ecosystem revealed by meta-and proteo-genomics. ISME J. 2011, 5, 1735-1747.

(28) Stroud, J. L.; Khan, M. A.; Norton, G. J.; Islam, M. R.; Dasgupta, T.; Zhu, Y. G.; Price, A. H.; Meharg, A. A.; McGrath, S. P.; Zhao, F. J. Assessing the labile arsenic pool in contaminated paddy soils by isotopic dilution techniques and simple extractions. Environ. Sci. Technol. 2011, 45 (10), 4262-4269.

(29) Yang, X. R.; Li, H.; Nie, S. A.; Su, J. Q.; Weng, B. S.; Zhu, G. B.; Yao, H. Y.; Gilbert, J. A.; Zhu, Y. G. Potential Contribution of Anammox to Nitrogen Loss from Paddy Soils in Southern China. Appl. Environ. Microbiol. 2015, 81, 938-947.

(30) Yamamura, S.; Watanabe, K.; Suda, W.; Tsuboi, S.; Watanabe, M. Effect of antibiotics on redox transformations of arsenic and diversity of arsenite-oxidizing bacteria in sediment microbial communities. Environ. Sci. Technol. 2013, 48 (1), 350-357.

(31) Mumford, A. C.; Barringer, J. L.; Benzel, W. M.; Reilly, P. A.; Young, L. Microbial transformations of arsenic: mobilization from glauconitic sediments to water. Water Res. 2012, 46 (9), 2859-2868.

(32) Ohtsuka, T.; Yamaguchi, N.; Makino, T.; Sakurai, K.; Kimura, K.; Kudo, K.; Homma, E.; Dong, D. T.; Amachi, S. Arsenic dissolution from Japanese paddy soil by a dissimilatory arsenate-reducing bacterium Geobacter sp. OR-1. Environ. Sci. Technol. 2013, 47 (12), $6263-6271$.

(33) Upadhyaya, G.; Clancy, T. M.; Brown, J.; Hayes, K. F.; Raskin, L. Optimization of arsenic removal water treatment system through characterization of terminal electron accepting processes. Environ. Sci. Technol. 2012, 46 (21), 11702-11709. 
(34) Rhine, E. D.; Garcia-Dominguez, E.; Phelps, C. D.; Young, L. Y. Environmental microbes can speciate and cycle arsenic. Environ. Sci. Technol. 2005, 39 (24), 9569-9573.

(35) Liu, W. J.; Zhu, Y. G.; Hu, Y.; Williams, P. N.; Gault, A. G.; Meharg, A. A.; Charnock, J. M.; Smith, F. A. Arsenic sequestration in iron plaque, its accumulation and speciation in mature rice plants (Oryza sativa L.). Environ. Sci. Technol. 2006, 40 (18), 5730-5736.

(36) Seyfferth, A. L.; Webb, S. M.; Andrews, J. C.; Fendorf, S. Arsenic localization, speciation, and co-occurrence with iron on rice (Oryza sativa L.) roots having variable Fe coatings. Environ. Sci. Technol. 2010, 44 (21), 8108-8113.

(37) Bhattacharjee, H.; Rosen, B. P. Arsenic metabolism in prokaryotic and eukaryotic microbes. In Molecular Microbiology of Heavy Metals; Springer-Verlag Press: Berlin, Germany, 2007; pp 371406.

(38) Hoeft, S. E.; Kulp, T. R.; Stolz, J. F.; Hollibaugh, J. T.; Oremland, R. S. Dissimilatory arsenate reduction with sulfide as electron donor: Experiments with mono lake water and isolation of strain MLMS-1, a chemoautotrophic arsenate respirer. Appl. Environ. Microbiol. 2004, 70 (5), 2741-2747.

(39) Héry, M.; van Dongen, B. E.; Gill, F.; Mondal, D.; Vaughan, D. J.; Pancost, R. R.; Polya, D. A.; Lloyd, J. R. Arsenic release and attenuation in low organic carbon aquifer sediments from West Bengal. Geobiology 2010, 8, 155-168.

(40) Islam, F. S.; Gault, A. G.; Boothman, C.; Polya, D. A.; Charnock, J. M.; Chatterjee, D.; Lloyd, J. R. Role of metal-reducing bacteria in arsenic release from Bengal delta sediments. Nature 2004, 430, 68-71.

(41) Knief, C.; Delmotte, N.; Chaffron, S.; Stark, M.; Innerebner, G.; Wassmann, R.; von Mering, C.; Vorholt, J. A. Metaproteogenomic analysis of microbial communities in the phyllosphere and rhizosphere of rice. ISME J. 2012, 6 (7), 1378-1390.

(42) Bentley, R.; Chasteen, T. G. Microbial methylation of metalloids: arsenic, antimony, and bismuth. Microbiol. Mol. Biol. Rev. 2002, 66 (2), 250-271.

(43) Mestrot, A.; Feldmann, J.; Krupp, E. M.; Hossain, M. S.; Roman-Ross, G.; Meharg, A. A. Field fluxes and speciation of arsines emanating from soils. Environ. Sci. Technol. 2011, 45 (5), 1798-1804.

(44) Lomax, C.; Liu, W. J.; Wu, L.; Xue, K.; Xiong, J.; Zhou, J.; McGrath, S. P.; Meharg, A. A.; Miller, A. J.; Zhao, F. J. Methylated arsenic species in plants originate from soil microorganisms. New. Phytol. 2012, 193 (3), 665-672.

(45) Chilvers, D.; Peterson, P. Global cycling of arsenic. Lead, Mercury, Cadmium, and Arsenic in the Environment; John Wiley \& Sons Press: New York, 1987; pp 279-301.

(46) Woolson, E. A. Generation of alkylarsines from soil. Weed Sci. 1977, 25 (5), 412-416.

(47) Lami, R.; Jones, L. C.; Cottrell, M. T.; Lafferty, B. J.; GinderVogel, M.; Sparks, D. L.; Kirchman, D. L. Arsenite modifies structure of soil microbial communities and arsenite oxidization potential. FEMS Microbiol. Ecol. 2013, 84 (2), 270-279.

(48) Rousk, J.; Bååth, E.; Brookes, P. C.; Lauber, C. L.; Lozupone, C.; Caporaso, J. G.; Knight, R.; Fierer, N. Soil bacterial and fungal communities across a pH gradient in an arable soil. ISME J. 2010, 4 (10), 1340-1351.

(49) Huang, H.; Jia, Y.; Sun, G. X.; Zhu, Y. G. Arsenic speciation and volatilization from flooded paddy soils amended with different organic matters. Environ. Sci. Technol. 2012, 46 (4), 2163-2168.

(50) Wang, X. J.; Chen, X. P.; Yang, J.; Wang, Z. S.; Sun, G. X. Effect of microbial mediated iron plaque reduction on arsenic mobility in paddy soil. J. Environ. Sci. 2009, 21 (11), 1562-1568. 